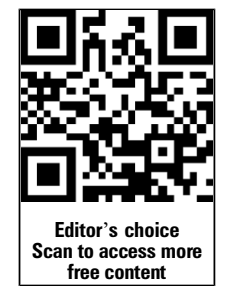

free content
${ }^{1}$ Department of Ophthalmology, Leiden University Medical Centre, Leiden, The Netherlands ${ }^{2}$ Department of Ophthalmology, Maxima Medical Centre, Veldhoven, The Netherlands ${ }^{3}$ Department of Ophthalmology, VU Medical Centre, Amsterdam, The Netherlands

${ }^{4}$ Department of Neonatology, St Antonius, Nieuwegein, The Netherlands

${ }^{5}$ Department of Ophthalmology, Amphia Hospital, Breda,

The Netherlands

${ }^{6}$ Department for Health Evidence, Radboud University Nijmegen Medical Centre, Nijmegen, The Netherlands ${ }^{7}$ Department of Ophthalmology, Sophia Children's HospitalErasmus MC, Rotterdam, The Netherlands

${ }^{8}$ Department of Neonatology, Wilhelmina Children's HospitalUniversity Medical Centre, Utrecht, The Netherlands

\section{Correspondence to} Dr A J van Sorge, Department of Ophthalmology, Leiden University Medical Centre, PO Box 9600, Leiden 2300 RC, The Netherlands: a.j.van_sorge@lumc.nl

Received 15 January 2013 Revised 28 May 2013 Accepted 17 June 2013 Published Online First 3 July 2013

\footnotetext{
To cite: van Sorge AJ, Schalij-Delfos NE, Kerkhoff $\mathrm{FT}$, et al. $\mathrm{Br} \mathrm{J}$ Ophthalmol 2013;97: 1143-1147.
}

\title{
Reduction in screening for retinopathy of prematurity through risk factor adjusted inclusion criteria
}

\author{
A J van Sorge, ${ }^{1} \mathrm{~N}$ E Schalij-Delfos, ${ }^{1} \mathrm{~F}$ T Kerkhoff, ${ }^{2} \mathrm{~L}$ J van Rijn, ${ }^{3}$ \\ $\mathrm{J} \mathrm{L}$ A M van Hillegersberg, ${ }^{4}$ I L A van Liempt, ${ }^{5} \mathrm{P} G \mathrm{M}$ Peer, ${ }^{6} \mathrm{H}$ J Simonsz, ${ }^{7}$ \\ J U M Termote ${ }^{8}$
}

\section{ABSTRACT}

Aims To develop a new national screening guideline for retinopathy of prematurity (ROP).

Methods Included were infants of the 2009 prospective ROP inventory in The Netherlands with gestational age $(G A)<32$ weeks and/or birth weight $(B W)<1500 \mathrm{~g}$. Five models were studied, based on GA and BW in combination with no, one or a set of five risk factors for ROP. Risk factors were determined by logistic regression. In MEDLINE and EMBASE, additional risk factors were searched. A precondition was that no infants with severe ROP would be missed. Receiver operating characteristic curves or classical measures were used to determine diagnostic accuracy.

Results The model including all infants with severe ROP comprised screening of infants with $G A<30$ weeks and/or BW $<1250 \mathrm{~g}$ and a selection of infants with GA 30-32 weeks and/or BW 1250-1500 g, with at least one of the following risk factors: artificial ventilation $(\mathrm{AV})$, sepsis, necrotising enterocolitis (NEC), postnatal glucocorticoids or cardiotonica. This model would not detect $4.8 \%(95 \% \mathrm{Cl} 2.5 \%$ to $8.0 \%)$ of infants with mild ROP and would reduce infants eligible for screening by $29 \%$.

Conclusions In The Netherlands, screening may be safely reduced using a new guideline based on GA, BW, $\mathrm{AV}$, sepsis, NEC, postnatal glucocorticoids and cardiotonica.

\section{INTRODUCTION}

Retinopathy of prematurity (ROP) is a serious vasoproliferative disease of the retina of the very premature infant, which may lead to visual impairment or blindness.

Treatment is possible and laser therapy is still preferable. As treated eyes have a better visual outcome than untreated eyes, timely detection of ROP through screening is important. ${ }^{1}$ Different screening criteria are used worldwide and depend mainly on national incidences of ROP, which in their turn mainly depend on socioeconomic factors and local neonatal care. However, highly developed countries vary in their criteria also. The current UK guideline recommends screening of all infants with a birth weight $(\mathrm{BW}) \leq 1500 \mathrm{~g}$ or a gestational age (GA) $\leq 31$ weeks. $^{2}$ The American guideline advises screening all infants with a BW $<1500 \mathrm{~g}$ or a GA $\leq 30$ weeks and selected infants between $1500 \mathrm{~g}$ and $2000 \mathrm{~g}$ with an unstable course. ${ }^{3}$ The screening criterion of Sweden is GA $\leq 31$ weeks. ${ }^{4}$ The Dutch guideline dating from 1997 advises screening infants with a BW $<1500 \mathrm{~g}$ and/or a GA $<32$ weeks or preterm infants treated $\geq 3$ days with $\geq 40 \%$ oxygen. ${ }^{5}$ These guidelines have in common that they screen infants with GA $\leq 30$ weeks, and most screen infants with GA 30-32 weeks. In the past decade, the incidence of ROP has altered. In Central Netherlands, a significant decrease in the incidence of overall and severe ROP was seen in infants with BW $<1000 \mathrm{~g}$ in the period 20012005 compared with 1991-1995. ${ }^{6}$ Also, Tan et al found a reduction in the incidence of severe ROP in southeast Scotland from 1990 to 2009.

As screening for ROP is uncomfortable for the neonate, and costly and time consuming for the ophthalmologist, the aim of this study was to evaluate whether our inclusion criteria for screening could be reduced on the condition that no infant with severe ROP would be missed.

\section{METHODS}

The study group consisted of patients included in the NEDROP study, a prospective inventory of ROP in preterm infants born in The Netherlands in 2009. Paediatricians and neonatologists of the 103 Dutch hospitals involved in the care of premature infants reported all infants eligible for ROP screening according to the national guideline to the study centre. ${ }^{5}$ Ophthalmologists reported all infants actually screened for ROP, any ROP classification, presence of 'plus disease', screening schedule and treatment. The NEDROP study was approved by the institutional review board (medical ethics committee of Leiden University Medical Centre, The Netherlands). To extend the clinical data of the study infants, coupling to the national perinatal registry (PRN) was performed. This database contains data of more than $95 \%$ of all infants with GA $<32$ weeks and/or BW $<1500$ g. $^{8}$ Because of the lack of data for older and heavier infants, only infants with GA $<32$ weeks and/or BW $<1500 \mathrm{~g}$ screened for ROP were included. Infants were categorised into two groups: infants with GA $<30$ weeks and/or BW $<1250 \mathrm{~g}$ (high risk (HR) group) and infants with GA 30-32 weeks and/or BW 1250-1500 g who do not fit into the HR group (moderate risk (MR) group). Clinical data, such as infant respiratory distress syndrome (IRDS), bronchopulmonary dysplasia (BPD), sepsis, intra-/ periventricular haemorrhage (IVH/PVH), necrotising enterocolitis (NEC) and pre- and postnatal 
glucocorticoids were classified according to the definitions of the PRN. ${ }^{8}$ In those cases where one or more characteristics were not recorded, we considered these data as missing.

ROP was classified following the International Classification of ROP. ${ }^{9}$ ROP was categorised as mild (stages 1-2) or severe (stages 3-5).

A thorough search of the literature for risk factors of ROP was performed in MEDLINE and EMBASE from 2006 to December 2011 using the search terms ROP, risk factor(s), prematurity, screening and guideline. Papers were limited to English, Dutch, French and German language. Reference lists of selected manuscripts were examined for additional relevant publications. Only those factors registered in our PRN, easily accessible and available at the time of the first screening were selected.

To improve the efficacy of our current screening programme, several models were studied and compared with regards to reduction in the number of infants to be screened and the number of infants with ROP that would not have been detected on the precondition that no infant with severe ROP would be missed. A second precondition was that the model should be easy to use clinically and would be implementable. First we investigated if screening could be reduced to infants in the HR group (model 1). Secondly, a prediction model for all infants of the study group was developed, based on GA and BW (model 2 ). Then one additional risk factor was added to the model based on GA and BW for all study group infants (model 3). Risk factors investigated were pre- and postnatal glucocorticoids, length of stay (LOS) on a neonatal intensive care unit (NICU), duration of artificial ventilation (AV), inhaled nitric oxide (iNO), gender, sepsis, IVH/PVH and NEC. In model 4 we investigated combining screening of the HR group with infants in the MR group with one specific risk factor, apart from GA and BW. Therefore, for infants in the MR group, a model based on BW, GA and one additional risk factor was developed. Risk factors included were those of model 2 , as well as patent ductus arteriosus, IRDS, BPD, supplemental oxygen, cardiotonica and erythrocyte transfusions. In model 5, screening of the HR group was combined with screening of infants in the MR group with at least one risk factor of a selected set of risk factors, including $\mathrm{AV}$, postnatal glucocorticoids, cardiotonica, sepsis and NEC.

\section{Statistical analysis}

To calculate the probability that a child will develop mild or severe ROP based on GA or BW, a multinomial logistic regression model was applied.

Additional risk factors for the development of ROP were explored with logistic regression. As some of the data consist of observations on multiple births, risk factors and probability of ROP for these neonates will be correlated. To take into account this dependency of the data, a generalised estimating equation approach was used to estimate the coefficients of the logistic regression model.

For each risk factor, a logistic regression model, including GA and BW, was explored. The ability of the model to discriminate between no ROP and ROP was quantified by the area under the receiver operating characteristic curve (AUC). For a model to be clinically useful, the predicted probabilities have to be low for children who do not develop ROP, intermediate for children with mild ROP and high for children with severe ROP. This was assessed graphically by plotting histograms of the predicted probabilities in the three groups.

Classic measures for the diagnostic accuracy of model 1 and model 5 were calculated with 95\% CIs.

\section{RESULTS}

Neonatologists and paediatricians reported 2193 infants, of whom $160(7.3 \%)$ died before screening was completed. Of the remaining 2033 infants, 1688 (83\%) were screened for ROP, of whom 1561 infants had GA $<32$ weeks and/or BW $<1500 \mathrm{~g}$. After coupling to the PRN, 1380 infants were eligible to enter the study $(81.8 \%$ of the screened population). Among these infants were 352 multiple births to 173 mothers. Infants had a median GA of 29.8 weeks and median BW was $1260 \mathrm{~g}$. Clinical data are shown in table 1 .

Table 1 Clinical characteristics of the study group, and incidence of (severe) retinopathy of prematurity for each characteristic

\begin{tabular}{|c|c|c|c|}
\hline Characteristic & Study group (\%) & Infants with ROP $(\%)^{*}$ & Infants with severe ROP $(\%)^{*}$ \\
\hline No & 1380 & $302(21.9)$ & $29(2.1)$ \\
\hline Median gestational age (weeks) $\left(\mathrm{P}_{25}-\mathrm{P}_{75}\right)$ & $29.8(28.1-31.1)$ & $28.0(26.4-29.4)$ & $26.3(25.4-27.0)$ \\
\hline Median birth weight $(g)\left(P_{25}-P_{75}\right)$ & $1260(1020-1500)$ & $950(780-1212)$ & $890(730-1060)$ \\
\hline Extremely low birth weight $(<1000 \mathrm{~g})$ & $345(25.0)$ & $178(51.6)$ & $6(1.7)$ \\
\hline Small for gestational age $\left(<\mathrm{P}_{10}\right)$ & $106(7.7)$ & $25(23.6)$ & $1(0.9)$ \\
\hline Female gender & $527(38.2)$ & $108(20.5)$ & $13(2.5)$ \\
\hline AV & $529(38.3)$ & $167(31.6)$ & $20(3.8)$ \\
\hline Supplemental $\mathrm{O}_{2}$ administration & $694(50.3)$ & $186(26.8)$ & $22(3.2)$ \\
\hline NICU admission & $1048(75.9)$ & $228(21.8)$ & $21(2.0)$ \\
\hline BPD & $65(4.7)$ & $38(58.5)$ & $8(12.3)$ \\
\hline Sepsis & $404(29.3)$ & $128(31.7)$ & $15(3.7)$ \\
\hline $\mathrm{IVH} / \mathrm{PVH}$ & $207(15.0)$ & $64(30.9)$ & $8(3.9)$ \\
\hline iNO & $23(1.7)$ & $11(47.8)$ & $2(8.7)$ \\
\hline Prenatal glucocorticoïds & $622(45.1)$ & $122(19.6)$ & $11(1.8)$ \\
\hline Postnatal glucocorticoïds & $78(5.7)$ & $45(57.7)$ & $14(17.9)$ \\
\hline IRDS & $663(48.0)$ & $177(26.7)$ & $20(3.0)$ \\
\hline NEC with perforation & $27(2.0)$ & $17(63.0)$ & $5(18.5)$ \\
\hline Erythrocyte transfusion & $549(39.8)$ & $186(33.9)$ & $20(3.6)$ \\
\hline
\end{tabular}


Figure 1 Predicted probability of no, mild and severe retinopathy of prematurity (ROP) in relation to gestational age for preterm infants born in 2009 in The Netherlands. Access the article online to view this figure in colour.

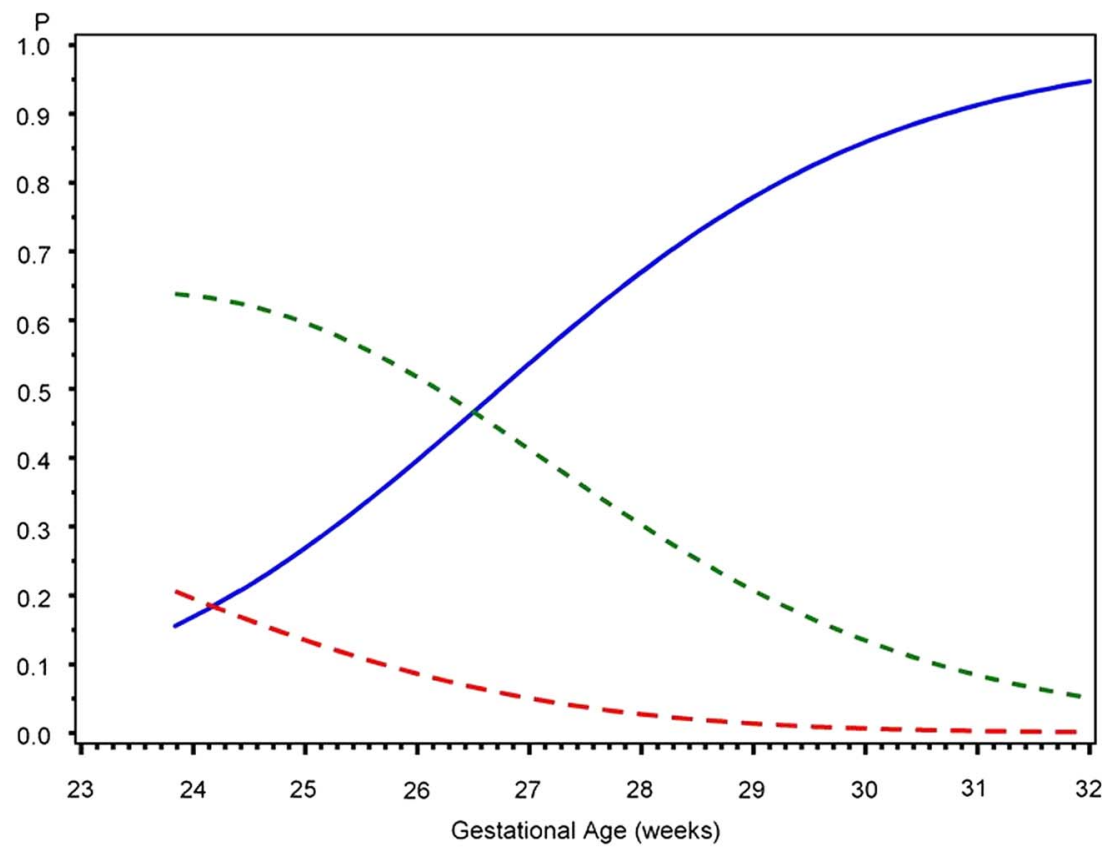

ROP - no - - m mild - - severe

In model 2, a prediction model for all infants of the study group was developed based on GA and BW. GA and BW were the most important risk factors for ROP in our study group (GA, $\mathrm{p}<0.0001$; BW, $\mathrm{p}<0.0001)$. The AUC for this model was 0.80 . Figure 2 shows the predicted probability of ROP using this model.

For infants without ROP, prediction was good, as the predicted probability was $\leq 0.20$ for $71.7 \%$ of infants with no ROP. For patients with mild and severe ROP, prediction was less accurate. Only $36.2 \%$ of infants with mild ROP had a predicted probability between 0.35 and 0.65 , and only $24.1 \%$ of infants with severe ROP had a predictive probability $\geq 0.70$.
Figure 2 Predicted probability of retinopathy of prematurity (ROP) based on gestational age and birth weight for infants in the study group classified by observed ROP. Access the article online to view this figure in colour.

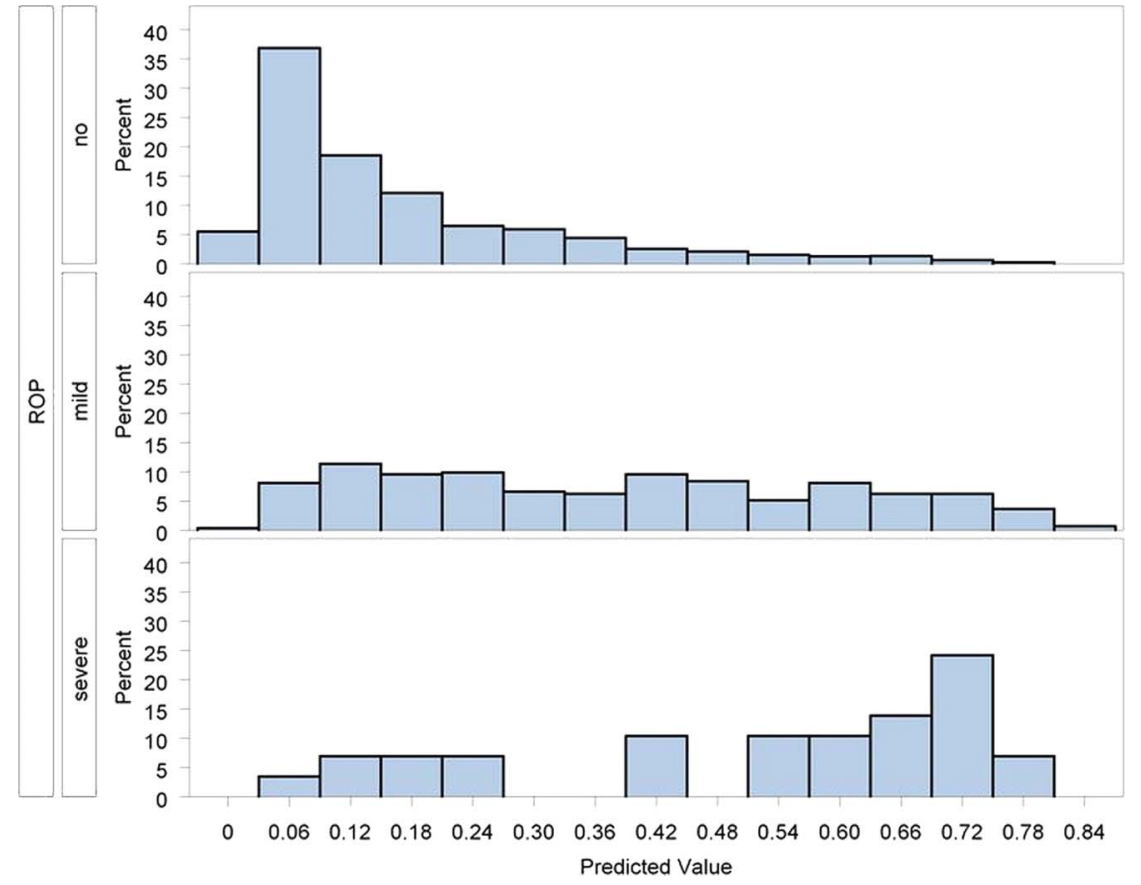


In model 3, the following risk factors were added to GA and BW, one at a time: pre- and postnatal glucocorticoïds, LOS on NICU, duration of AV, iNO, gender, sepsis, IVH/PVH and NEC. None of these risk factors resulted in improvement in AUC.

In the MR group $(n=509), 27$ infants $(5.3 \%)$ developed mild and one infant $(0.2 \%)$ severe ROP. The AUC of the model, based only on GA and BW, was moderate in the MR group (0.69). Addition of any of the following risk factors gave no improvement in AUC: pre- and postnatal glucocorticoids, gender, sepsis, IVH/PVH, patent ductus arteriosus, IRDS, BPD, duration of AV, LOS on NICU, iNO, supplemental oxygen, cardiotonica, NEC and erythrocyte transfusion (range of AUCs 0.68-0.72) (model 4).

For model 5, a set of risk factors was selected. Of the risk factors found through the literature search, AV, postnatal glucocorticoïds, cardiotonica, sepsis and NEC met our selection conditions. Of the 509 infants in the MR group, 107 needed $\mathrm{AV}$, four postnatal glucocorticoïds, 28 had cardiotonica, 105 had sepsis and 10 had NEC. No risk factors were found in 271 infants (53.2\%) and $\geq 1$ risk factors in 177 infants (34.8\%); data for 61 infants $(12.0 \%)$ were incomplete.

The true positive rate (TPR) of model 5 was $0.26(289 / 1109)$ (95\% CI 0.23 to 0.29 ) and the true negative rate (TNR) was 0.95 (258/271) (95\% CI 0.91 to 0.98$)$, assuming that children with missing data were also screened. The TPR of this model was low but no infant with severe ROP, and only 13/273 infants with mild ROP, were missed. Introduction of model 5 would result in exclusion from screening of at least $19.6 \%(271 / 1380)$ of infants in the study group, again assuming that infants with missing data are also screened. In comparison with the old guideline, this model would have resulted in a reduction of $29.0 \%(452 / 1561)$ of infants and $21.2 \%(786 / 3705)$ of examinations (table 2$)$.

\section{DISCUSSION}

The aim of this study was to develop a new guideline for ROP screening in The Netherlands, on condition that restriction of the inclusion criteria for screening would not lead to infants with severe ROP being missed. For development of the guideline, a predictive ROP model was evolved for which ROP and clinical data for all infants with GA $<32$ weeks and/or BW $<1500 \mathrm{~g}$ born in The Netherlands in 2009 and completely screened for ROP were used. Five different models were investigated. The model which included all infants with GA $<30$ weeks and/or BW $<1250 \mathrm{~g}$ as well as infants with GA $30-32$ weeks and/or BW $1250-1500 \mathrm{~g}$ with one or more of the risk factors AV, NEC, sepsis, postnatal glucocorticoids or cardiotonica, predicted the risk of ROP best (TPR 0.26 and TNR 0.95). Using this model, no infant with severe ROP was missed and $4.8 \%$ with mild ROP were missed. We performed a literature search to find the most established risk factors for ROP and compared these with those available in our database and found in our study. Apart from being easily accessible, they should be available before the age of 5 weeks postpartum, as this is the time at which ROP screening for many infants already starts. Seiberth et al, Karna et al and Hoogerwerf et al demonstrated that duration of $\mathrm{AV}$ is a risk factor for (severe) ROP. ${ }^{6} 10{ }^{11}$ Treatment with dopamine was a risk factor for (severe) ROP in studies of Mizoguchi et al and Liu et al. ${ }^{12}{ }^{13}$ Hoogerwerf et al, Smolking et al and the systematic review of the Cochrane database showed that (late) treatment with postnatal steroids is a risk factor for ROP. ${ }^{614}{ }^{15}$ Lee, Chen et al, Weintraub et al and Jensen et al demonstrated perinatal infection/inflammation or sepsis to be a risk factor for (severe) ROP. $^{16-19}$ Arrøe proved this for NEC. ${ }^{20}$

Most national ROP guidelines are based on GA and/or BW, the major risk factors for ROP. Another important risk factor, postnatal illness, is seldom considered. Recently, four ROP screening models based on postnatal course were developed: WINROP, ROPscore, cumulative illness severity and a model based on Clinical Risk Index for Babies score, multiple birth, race and gender. ${ }^{21-24}$ All predicted proliferative ROP requiring treatment appropriately.

The former mentioned models could not be applied to our study as the PRN contains only limited information. Due to privacy regulations, data were also not accessible. In order to reflect the postnatal course, we added the risk factors $\mathrm{AV}$, cardiotonica, postnatal steroids, sepsis and NEC, to GA and BW as selection criteria in our new screening guideline.

Our new guideline has two important advantages. First, compared with the old one, this model reduced the number of infants who required eye examinations by $29 \%$, whereas $4.8 \%$ of infants with mild ROP and no infants with severe ROP would have gone undetected. This corresponds with a reduction of $21 \%$ of fundoscopies. As screening is stressful for the neonate, this decrease is important. On the other hand, we should also consider that screening can be made less uncomfortable for the infant and more efficient for the ophthalmologist by swaddling of the infant, use of a pacifier and/or sucrose, and by good positioning of the infant.

Secondly, our guideline takes several pathological conditions into account that might occur within the first 4 weeks of life and predispose to the onset of ROP.

Our model also has two disadvantages. First, a small percentage of infants with mild ROP will go undiagnosed. Secondly, for the group of infants with GA 30-32 weeks and/or BW 1250-1500 g, who do not fit into the HR group, our model contains five items which have to be assessed. This implies extra work for the attending neonatologist or paediatrician. All items are, however, easily accessible and should be present in the letter of transfer, if patients are already discharged from the NICU before the first screening. If risk factors are not properly

Table 2 Reduction in number of infants that need to be screened and in number of fundus examinations for different screening criteria in comparison with the old guideline

\begin{tabular}{|c|c|c|c|c|}
\hline Inclusion criteria & Infants (n) & Examinations (n) & $\begin{array}{l}\text { Reduction in } \\
\text { infants (\%) }\end{array}$ & $\begin{array}{l}\text { Reduction in } \\
\text { examinations (\%) }\end{array}$ \\
\hline$<32$ weeks and/or $<1500 \mathrm{~g}$ & 1561 & 3705 & & \\
\hline$<32$ weeks and/or $<1500 \mathrm{~g}$ (study group coupled to PRN) & 1380 & 3339 & 11.6 & 9.9 \\
\hline$<30$ weeks and/or $<1250 \mathrm{~g}$ (HR group) & 871 & 2539 & 44.2 & 31.5 \\
\hline $\begin{array}{l}<30 \text { weeks and/or }<1250 \mathrm{~g} \text { and } 30-32 \text { weeks and/or } 1251-1500 \mathrm{~g} \text { with } \\
\geq 1 \text { risk factor or unrecorded risk factors }\end{array}$ & 1109 & 2919 & 29.0 & 21.2 \\
\hline
\end{tabular}


recorded, the old criteria (GA $<32$ weeks and/or BW $<1500$ g) should be used. External validation is essential for a new guideline. ${ }^{25}$ Validation of the new guideline is currently underway in The Netherlands since September 2012. Future studies will be necessary for monitoring the accuracy of the model, as it was based on an ROP inventory study in 2009.

In conclusion, in The Netherlands, ROP screening may be reduced by adding risk factors representative for each patient's postnatal course to the well known risk factors of GA and BW. Although this implies an additional effort on the part of the neonatologist, it will reduce the number of infants exposed to these stressful examinations and will allow ophthalmologists to focus on those infants with the highest risk for ROP. Monitoring of the new guideline is necessary.

Acknowledgements We thank W Lemmens from the Department for Health Evidence, Radboud University Nijmegen Medical Centre, Nijmegen, for data management and statistical analyses.

Contributors All authors took part in the development of these new screening criteria. AJvS, JUMT and NES-D wrote the manuscript and the remaining authors gave their feedback and modifications. Data calculations were done by PGMP, as were all of the statistical analyses.

Funding This study was supported by an unrestricted grant from the ODAS Foundation, Delft, The Netherlands.

\section{Competing interests None.}

Ethics approval The study was approved by the institutional review board (medical ethics committee of Leiden University Medical Centre, The Netherlands).

Provenance and peer review Not commissioned; externally peer reviewed.

\section{REFERENCES}

1 Good WV, Hardy RJ, Dobson V, et al. Final visual acuity results in the early treatment for retinopathy of prematurity study. Arch Ophthalmol 2010;128:663-71.

2 Wilkinson $A R$, Haines $L$, Head $K$, et al. UK retinopathy of prematurity guideline. Eye (Lond) 2009;23:2137-9.

3 Section on Ophthalmology American Academy of Pediatrics; American Academy of Ophthalmology; American Association for Pediatric Ophthalmology and Strabismus. Screening examination of premature infants for retinopathy of prematurity. Pediatrics 2006;117:572-6.

4 Larsson $\mathrm{E}$, Holmstrom $\mathrm{G}$. Screening for retinopathy of prematurity: evaluation and modification of guidelines. Br J Ophthalmol 2002;86:1399-402.

5 Schalij-Delfos NE, Zijlmans BL, Wittebol-Post D, et al. Screening for retinopathy of prematurity: do former guidelines still apply? J Pediatr Ophthalmol Strabismus 1996;33:35-8.
6 Hoogerwerf A, Schalij-Delfos NE, van Schooneveld MJ, et al. Incidence of retinopathy of prematurity over the last decade in the Central Netherlands. Neonatology 2010;98:137-42.

7 Tan SZ, Dhaliwal C, Becher JC, et al. Trends in the incidence of retinopathy of prematurity in Lothian, south-east Scotland, from 1990 to 2009. Arch Dis Child Fetal Neonatal Ed 2012;97:F310-11.

8 http://www.perinatreg.nl/wat_wordt_geregistreerd. 2011.

9 International Committee for the Classification of Retinopathy of Prematurity. The International Classification of Retinopathy of Prematurity revisited. Arch Ophthalmol 2005;123:991-9.

10 Seiberth $\mathrm{V}$, Linderkamp 0 . Risk factors in retinopathy of prematurity. a multivariate statistical analysis. Ophthalmologica 2000;214:131-5.

11 Karna P, Muttineni J, Angell L, et al. Retinopathy of prematurity and risk factors: a prospective cohort study. BMC Pediatr 2005:5:18

12 Mizoguchi MB, Chu TG, Murphy FM, et al. Dopamine use is an indicator for the development of threshold retinopathy of prematurity. $\mathrm{Br} J$ Ophthalmol 1999;83:425-8

13 Liu PM, Fang PC, Huang $C B$, et al. Risk factors of retinopathy of prematurity in premature infants weighing less than $1600 \mathrm{~g}$. Am J Perinatol 2005;22:115-20.

14 Smolkin T, Steinberg M, Sujov P, et al. Late postnatal systemic steroids predispose to retinopathy of prematurity in very-low-birth-weight infants: a comparative study. Acta Paediatr 2008;97:322-6.

15 Halliday HL, Ehrenkranz RA, Doyle LW. Late (>7 days) postnatal corticosteroids for chronic lung disease in preterm infants. Cochrane Database Syst Rev 2009;1: CD001145.

16 Lee J, Dammann 0. Perinatal infection, inflammation, and retinopathy of prematurity. Semin Fetal Neonatal Med 2012;17:26-9.

17 Chen M, Citil A, McCabe F, et al. Infection, oxygen, and immaturity: interacting risk factors for retinopathy of prematurity. Neonatology 2011;99:125-32.

18 Weintraub Z, Carmi N, Elouti $\mathrm{H}$, et al. The association between stage 3 or higher retinopathy of prematurity and other disorders of prematurity. Can J Ophthalmol 2011:46:419-24.

19 Jensen AK, Ying GS, Huang J, et al. Thrombocytopenia and retinopathy of prematurity. J AAPOS 2011;15:e3-4.

20 Arroe M, Peitersen B. Retinopathy of prematurity: review of a seven-year period in a Danish neonatal intensive care unit. Acta Paediatr 1994;83:501-5.

21 Hellstrom A, Hard AL, Engstrom E, et al. Early weight gain predicts retinopathy in preterm infants: new, simple, efficient approach to screening. Pediatrics 2009;123: e638-45.

22 Eckert GU, Fortes Filho JB, Maia M, et al. A predictive score for retinopathy of prematurity in very low birth weight preterm infants. Eye (Lond) 2012;26:400-6.

23 Yang MB, Donovan EF, Wagge JR. Race, gender, and clinical risk index for babies (CRIB) score as predictors of severe retinopathy of prematurity. J AAPOS 2006;10:253-61

24 Hagadorn Jl, Richardson DK, Schmid CH, et al. Cumulative illness severity and progression from moderate to severe retinopathy of prematurity. J Perinatol 2007;27:502-9.

25 Bleeker SE, Moll HA, Steyerberg EW, et al. External validation is necessary in prediction research: a clinical example. J Clin Epidemiol 2003:56:826-32. 